Journal of Animal and Veterinary Advances 11 (6): 742-745, 2012

ISSN: $1680-5593$

(C) Medwell Journals, 2012

\title{
Detection Class I Integron of Multidrug Resistace Pathogenic Salmonella from Chicken in Hebei Province, China
}

\author{
${ }^{1}$ Qiuyue Wang, ${ }^{1}$ Qiumei Shi, ${ }^{1}$ Yanying Zhang, ${ }^{2}$ Wei Yao, ${ }^{1}$ Cuizhen Chen, \\ ${ }^{1}$ Guangping Gao, ${ }^{1}$ Donglin Zhang and ${ }^{1}$ Hai Fang \\ ${ }^{1}$ Key Laboratory of Preventive Veterinary Medicine of Hebei Province, \\ Hebei Normal University of Science and Technology, 066600 Qinhuangdao, China \\ ${ }^{2}$ Heibei Qinglong County Pingfangzi Junior Middle School, 066503 Qinhuangdao, China
}

\begin{abstract}
Antimicrobial resistance test of 59 Salmonella isolated from chicken was done by K-B method. The drug-resistance rate of oxacillin, tetracycline, erythromycin, penicillin $\mathrm{G}$, gentamicin was $74.6,64.4,52.5,47.5$ and $37.3 \%$, respectively. Most of the isolates were multidrug resistance and the rate was $81.36 \%(48 / 59)$. Among the total of the multidrug resistance isolates, the rate of resistance to three, four and five drugs was $45.76 \%$ (27/59), 23.73\% (14/59) and 11.86\% (7/59). PCR and DNA sequencing were used for screening and characterization of class I integrons. As a result, the detection rate of class I integron was 40.68\% (24/59) and among these 48 isolates which had been proven multidrug-resistant, the detection rate of class I integron was $50 \%$ (24/48). Class I integron PCR positive isolates rate of multidrug-resistance was $100 \%(24 / 24)$. Results show that the class I integron has the relationship with multidrug resistance.
\end{abstract}

$\underline{\text { Key words: Salmonella, class I integron, multidrug resistance, detection, multidrug, China }}$

\section{INTRODUCTION}

Avian Salmonellosis is a major bacteriosis in henneries causing deaths of chickens in great numbers, hampering the growth and development of chickens, increasing the possibility of contamination of eggs (Hall and Saito, 2008). Currently the control strategies of avian salmonellosis besides vaccines, antibiotics are mainly applied for disease treatment and prevention. However, due to the continual clinical using of antibiotics in large amount, antimicrobial drug resistance is an increasing problem in Salmonella with strains more and more commonly being isolated exhibiting Multidrug Resistance (MDR). Consequently the detection of Salmonella drug resistance is very important to prevent avian salmonellosis.

In the study of the mechanism of bacterial multidrug resistance, the presence of genes within integrons coding for antibiotic resistance and the association of antibiotic resistance phenotypes with the presence of integrons have been well documented (Maguire et al., 2001; Voetal., 2006).

Integrons are gene expression elements that play an important role in the recruitment of antimicrobial drug resistance determinants via site-specific recombination events catalyzed by the integron-encoded integrase (Guerra et al., 2000). Based on the sequence of the integrase genes, several classes of integrons have been described (Nogrady et al., 2005; Gebreyes et al., 2004).

Currently the detection of class I integron consist mainly in the amplification of its resistance gene cassette. Therefore, the aim of this study was to investigate the prevalence and characterize class I integron among Salmonella strains separated from chickens and to monitor the spread of the multidrug-resistant Salmonella strains. Analyze the relation between class I integron and Multiple Drug Resistance (MDR) in molecular level.

\section{MATERIALS AND METHODS}

Bacterial strains: A total of 59 Salmonella strains were isolated from chicken in Hebei province. The identification of each isolate as Salmonella was confirmed by performing PCR for the invA gene as described previously (Rao et al., 2008; Chiu and Ou, 1996) and serotyped by standard procedures. At last, all the Salmonella we have identified were further identified and confirmed in the professional laboratory of Salmonella, Chengdu Institute of Biological Products. Samples were kept in $-20^{\circ} \mathrm{C}$ for further study.

DNA extraction and amplification: DNA was extracted from the Salmonella isolates using the bacterial genomic

Corresponding Author: Hai Fang, Key Laboratory of Preventive Veterinary Medicine of Hebei Province, Hebei Normal University of Science and Technology, 066600 Qinhuangdao, China 
Table 1: Primers designed for this research

\begin{tabular}{lllr}
\hline Region or gene & Name & Sequence $\left(5^{\prime}-3^{\prime}\right)$ & Temp. $\left({ }^{\circ} \mathrm{C}\right)$ \\
\hline Integrase $\alpha$ & Int-F & TGCGTGTAAATCATCATCGTCGTAGAG & 56 \\
& Int-R & ACAGCTTACGAACCGAACAGGC & 56 \\
Variable segment & Var-F & CGATGTTGATGTTATGGAGCAG & 53 \\
& Var-F & ACTTGACCTGATAGTTGGCTGTG & 53 \\
$5^{\prime}-3^{\prime}$ conserved segment & $3^{\prime}-\mathrm{F}$ & CGCAATAGTTGGCGAAGTAATC & 56 \\
& $3^{\prime}-\mathrm{R}$ & GAAGAACCGCACAATCTCGTC & 56 \\
\hline
\end{tabular}

DNA Extraction kit (Takara, Dalian, China) and used as the template for the detection of integrons by PCR with primers. Three pairs of primers shown in Table 1 were designed according to the structure of class I integron. Amplification was performed using the following temperature profile: an initial denaturation step at $94^{\circ} \mathrm{C}$ for $5 \mathrm{~min}, 35$ cycles each at $94^{\circ} \mathrm{C}$ for $40 \mathrm{sec}$, primer annealing at different temperature for $40 \mathrm{sec}$ shown in Table 1 and an extension step at $72^{\circ} \mathrm{C}$ for $1 \mathrm{~min}$. The final extension time was $10 \mathrm{~min}$ at $72^{\circ} \mathrm{C}$. The amplified DNA products were analyzed by conventional $0.8 \%$ agarose gel electrophoresis in $1 \times \mathrm{TAE}$ (Tris-Acetate-EDTA) buffer at $120 \mathrm{~V}$. Purified integron DNA samples were sequenced using primers as mentioned in Shanghai Sangon Co., Ltd. and blast using the BLAST program available at the NCBI BLAST homepage (http://blast.ncbi.nlm.nih.gov/Blast. cgi).

Drug sensitive test: Antibiotic susceptibility testing of Salmonella isolates was carried out by the K-B method employing a replicator. Antimicrobial breakpoints were selected according to the National Committee for Clinical Laboratory Standards (NCCLS) (Wayne, 2001). Quality controls strains were Escherichia coli ATCC 25922, Staphylococcus aureus ATCC 29923 and Pseudomonas aeruginosa ATCC 27853. All plates were incubated for $18 \mathrm{~h}$ at $37^{\circ} \mathrm{C}$.

The antibiotics selected and breakpoints for resistance were as follows: ampicillin, amoxicillin, ceftriaxone, cefoperazone, cephalothin, cefazolin, cefepime, ceftazidime, ciprofloxacin, chloramphenicol, ciprofloxacin, gentamicin, tetracycline, oxacillin, ofloxacin, erythromycin, kanamycin, sulfisoxazole/trimethoprim, levofloxacin, cefuroxime sodium, chloramphenicol and piperacillin. These antibiotics slips were purchased from Beijin Temple of Heaven Biotechnology Technical Development Company.

\section{RESULTS}

Drug sensitive test: The drug resistance patterns of 59 strains Salmonella from chicken are shown in Table 2. High resistance rates in 59 strains of Salmonella were observed for oxacillin $74.6 \%$ (44/59), tetracycline $64.4 \%$ (38/59) and erythromycin 52.5\% (31/59), respectively. The resistance rates to penicillins (oxacillin, ampicillin,
Table 2: The 59 strains Salmonella isolates resistance rate

\begin{tabular}{|c|c|c|c|}
\hline Typing & Antibacterials & $\begin{array}{l}\text { Drug } \\
\text { resistant strain }\end{array}$ & $\begin{array}{c}\text { Resistance } \\
\text { rate }(\%)\end{array}$ \\
\hline \multirow[t]{5}{*}{ Penicillins } & Oxacillin & $44 / 59$ & 74.6 \\
\hline & Ampicillin & 15 & 25.4 \\
\hline & Piperacillin & 0 & 0.0 \\
\hline & Amoxicillin & 0 & 0.0 \\
\hline & Penicillin G & $28 / 59$ & 47.5 \\
\hline \multirow[t]{7}{*}{ Cephalosporins } & Cefoperazone & 0 & 0.0 \\
\hline & Cephalothin & 0 & 0.0 \\
\hline & Cefazolin & 0 & 0.0 \\
\hline & Cefepime & 0 & 0.0 \\
\hline & Ceftazidime & 0 & 0.0 \\
\hline & Cefuroxime sodium & 0 & 0.0 \\
\hline & Ceftriaxone & 0 & 0.0 \\
\hline \multirow[t]{2}{*}{ Aminoglycosides } & Kanamycin & 0 & 0.0 \\
\hline & Gentamicin & $22 / 59$ & 37.3 \\
\hline Tetracyclines & Tetracycline & $38 / 59$ & 64.4 \\
\hline Amphenicols & Chloramphenicol & $7 / 59$ & 11.9 \\
\hline Macrolides & Erythromycin & $31 / 59$ & 52.5 \\
\hline \multirow[t]{2}{*}{ Sulfonamides } & Sulfisoxazole/ & $11 / 59$ & 18.6 \\
\hline & Trimethoprim & - & - \\
\hline \multirow[t]{3}{*}{ Quinolones } & Ofloxacin & 0 & 0.0 \\
\hline & Levofloxacin & 0 & 0.0 \\
\hline & Ciprofloxacin & 0 & 0.0 \\
\hline
\end{tabular}

piperacillin, amoxicillin, penicillin G) ranged from $0.0 \sim 74.6 \%$ in those isolates. Except for penicillins and tetracyclines, high resistance rates $(37.3 \sim 52.5 \%)$ were observed for the other macrolides and aminoglycosides. The 59 strains Salmonella from chicken have the high susceptibility to cephalosporins (resistance rate $0.0 \%$ ) and quinolones $(0.0 \%)$.

Some isolates in the 59 strains from chicken were resistant to 8 species antibiotics at most. There were only 11 strains resistant to 2 species antibiotics and the others were multidrug resistance. Most of the isolates had the multidrug resistance pattern and the rate was $81.36 \%$ (48/59). Among the total of the multidrug resistance isolates, the rate of resistance to three, four and five drugs was $45.76 \%$ (27/59), 23.73\% (14/59) and $11.86 \%(7 / 59)$.

Amplification of integrase $\alpha$ and $5^{\prime}-3^{\prime}$ conserved segments: PCR and DNA sequencing were used for screening and characterization of class I integrons. A fragment of approximately 856 bp of integrase $\alpha$ gene was resulted by PCR amplification using primers Int-F and Int-R (Fig. 1). The PCR product were purified and used directly as templates for sequencing. Among these 59 isolates, integrase $\alpha$ segment had been detected from 24 strains. On the basis of the results of integrase $\alpha$ 


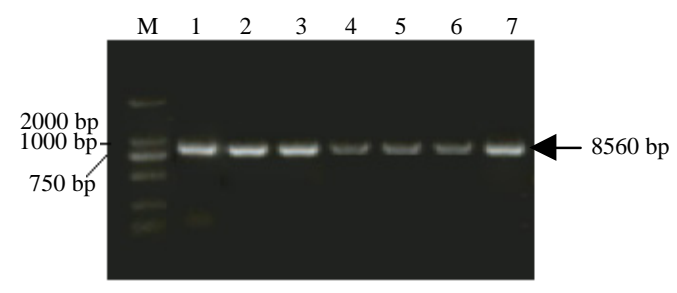

Fig. 1: PCR detection of integrase $\alpha$ gene from Salmonella isolates; M: DNA Marker DL2000; 1 7: Experiment strain

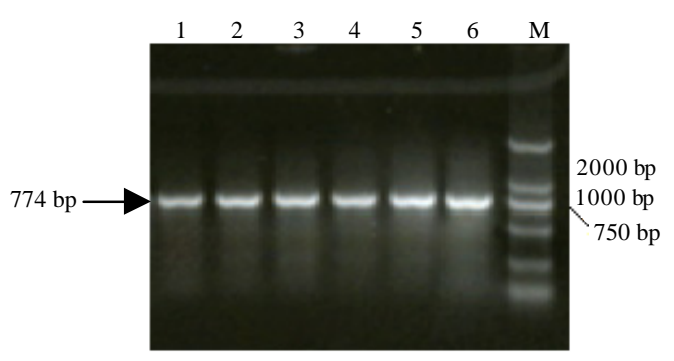

Fig. 2: PCR detection of 5'-3' conserved segments gene from Salmonella isolates; M: DNA Marker DL2000; 1 6: Experiment strain

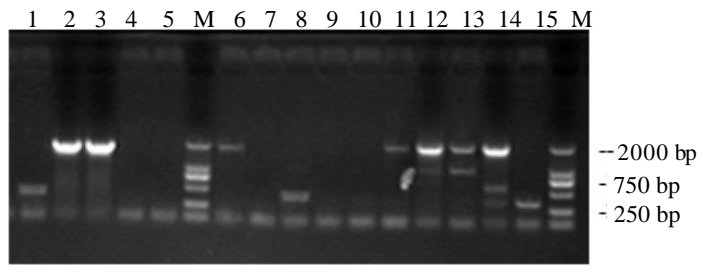

Fig. 3: PCR detection of variable segment in integrase-positive strains; $\mathrm{M}$ : DNA Marker DL2000; 1 15: The fragment of gene cassette ranged from $300-2000 \mathrm{bp}$

segment, the $5^{\prime}-3^{\prime}$ conserved segment were amplification using primers $3^{\prime}-\mathrm{F}$ and $3^{\prime}-\mathrm{R}$. All the strains containing integrase $\alpha$ segment proved positive. The segment of 774 bp was detected which corresponds to the expected size of the 5'-3' conserved gene (Fig. 2).

Amplification of variable segment: The researchers detected the variable segment in the integrase-positive strains using PCR amplification. Variable segments were found in $70.8 \%(17 / 24)$ of integrase-positive strains. With the variable region of the resistance genes carrying different type and different number, the amplified fragment size is also different. The segments different from 300-2000 bp were observed (Fig. 3).

Integron carriage and the association of resistance to antimicrobials: As a result, the detection rate of class I integron was $40.68 \%$ (24/59) and among these 48 isolates which had been proven multidrug-resistant, the detection rate of class I integron was 50\% (24/48). Class I integron PCR positive isolates rate of multidrug-resistance was $100 \%(24 / 24)$. Results show that the class I integron has the relationship with multidrug resistance. However, some isolates without integrons were also had the multidrug resistance pattern, most commonly resistance to tetracycline, oxacillin, ampicillin and gentamicin.

\section{DISCUSSION}

Many gene cassettes of integrons encoding antimicrobial resistance genes are found in gram-negative sbacteria. The horizontal transfer of integrons among bacteria plays an important role in the dissemination of antimicrobial resistance gene, resulting in the development of multidrug resistance (Yang et al., 2009). So, far 6 types of integrons are defined based on thire homology to the integrase proteins (Hall and Collis, 1995). Among those integrons, class I integrons are commonly observed in bacteria. Class I integrons are most commonly found in clinical isolates of gram-negative bacteria and $>100$ gene cassettes that confer resistance are known.

Among the total of 59 Salmonella strains from chicken in the study, the rate of class I integron PCR positive isolates was $40.68 \%$ (24/59), consistent with the reported $(59 \sim 70 \%)$. The results suggest that the presence of class I integron has significant effect on the rate of multidrug resistant isolates of Salmonella. In this study, class I integron positive isolates rate of multidrug-resistance was $100 \%(24 / 24)$ while the negative strains $68.57 \%(24 / 35)$. Multidrug-resistance rate of positive strains was significantly higher than the negative multidrug resistant strains. Results show that the class I integron has the obvious relationship with multidrug resistance.

In this experiment, variable segments were found in $70.8 \%(17 / 24)$ of integrase-positive strains. But the amplification of variable region from the other 7 strains which containing integrase $\alpha$ and $5^{\prime}-3^{\prime}$ conserved segments was negative. Possible reason is carrying empty gene cassette with the strains. Once the appropriate conditions or antibiotic selection pressure appeared, the empty gene cassette can be integrated showing drug resistance. Amplification results from the variable region above, the fragment of gene cassette ranged from 300-2000 bp. Previously reported in the literature to design conserved primers to amplify the variable region as the method of detection integron positive strains (Rao et al., 2008; Daly and Fanning, 2000; Guerra et al., 2000), amplification results from this study explained this method is not conducive to the detection of non typical class I integron. 


\section{CONCLUSION}

This study shows that the aggravating resistance of bacteria against antibiotics and increasing cases of MDR pose a fatal threat to the health of humans and animals. Theory of integron system offers a new direction for researching the mechanism of bacterial resistance (Guerra et al., 2000). At present, every class of antibiotic resistance gene cassettes has been found and all the resistance gene cassettes have been associated with antibiotics. Integration of integrons, resistance genes can be exchanged and with the transformation, transduction and conjugation of plasmids, drug resistance can spread widely among creatures. Since, bacterial resistance is a very complex process with many mechanisms, integron's effect on MDR is only one of them, the structure and function of integron-gene cassette system should be conducted on for further study. Researchers need to use the current drugs better and use the best and most affordable drugs available in order to prevent further resistance.

\section{ACKNOWLEDGEMENT}

This study partially supported by Hebebi Science and Technology Support Program (09220402D).

\section{REFERENCES}

Chiu, C.H. and J.T. Ou, 1996. Rapid identification of Salmonella serovars in feces by specific detection of virulence genes, invA and $\mathrm{spvC}$, by an enrichment broth culture-multiplex PCR combination assay. J. Clin. Microbiol., 34: 2619-2622.

Daly, M. and S. Fanning, 2000. Characterization and chromosomal mapping of antimicrobial resistance genes in Salmonella enterica serotype typhimurium. Applied Environ. Microbiol., 66: 4842-4848.

Gebreyes, W.A., S. Thakur, P.R. Davies, J.A. Funk and C. Altier, 2004. Trends in antimicrobial resistance, phage types and integrons among Salmonella serotypes from pigs, 1997-2000. J. Antimicrob. Chemother., 53: 997-1003.
Guerra, B., S. Soto, S. Cal and M.C. Mendoza, 2000. Antimicrobial resistance and spread of class 1 integrons among Salmonella serotypes. Antimicrob. Agents Chemother., 44: 2166-2169.

Hall, A.J. and E.K. Saito, 2008. Avian wildlife mortality events due to salmonellosis in the United States, 1985-2004. J. Wildlife Dis., 44: 585-593.

Hall, R.M. and C.M. Collis, 1995. Mobile gene cassettes and integrons: Capture and spread of genes by sitespecific recombination. Mol. Microbiol., 15: 593-600.

Maguire, A.J., D.F. Brown, J.J. Gray and U. Desselberger, 2001. Rapid screening technique for class 1 integrons in Enterobacteriaceae and nonfermenting gramnegative bacteria and its use in molecular epidemiology. Antimicrob. Agents Chemother., 45: 1022-1029.

Nogrady, N., I. Gado, A. Toth and J. Paszti, 2005. Antibiotic resistance and class 1 integron patterns of non-typhoidal human Salmonella serotypes isolated in Hungary in 2002 and 2003. Int. J. Antimicrob. Agents, 26: 126-132.

Rao, S., C.W. Maddox, P. Hoien-Dalen, S. Lanka and R.M. Weigel, 2008. Diagnostic accuracy of class 1 integron PCR method in detection of antibiotic resistance in Salmonella isolates from swine production systems. J. Clin. Microbiol., 46: 916-920.

Vo, A.T., E. van Duijkeren, A.C. Fluit, W.J. Wannet, A.J. Verbruggen, H.M. Maas and W. Gaastra, 2006. Antibiotic resistance, integrons and Salmonella genomic island 1 among non-typhoidal Salmonella serovars in The Netherlands. Int. J. Antimicrob. Agents, 28: 172-179.

Wayne, P.A., 2001. NCCLS. Performance standards for antimicrobial susceptibility testing. Eleventh International Supplement. Veterinary Antimicrobial Susceptibility Testing Document M31-A2. 21(1). www.clsi.org/source/orders/free/m100-s21.pdf.

Yang, C.M., M.F. Lin, C.H. Lin, Y.T. Huang, C.T. Hsu and M.L. Liou, 2009. Characterization of antimicrobial resistance patterns and integrons in human fecal Escherichia coli in Taiwan. Jpn. J. Infect. Dis., 62: 177-181. 